\title{
Temporal and Qualitative Differences in the Development of Allodynic Behaviors between Mice and Rats in a Peripheral Nerve Injury Model
}

\author{
Alexandra Sideris ${ }^{1 *}$, Monica Norcini1 ${ }^{1}$, Thomas J. J. Blanck ${ }^{1,2}$, Esperanza Recio-Pinto ${ }^{1,3}$ \\ ${ }^{1}$ Department of Anesthesiology, NYU Langone Medical Center, New York, USA \\ ${ }^{2}$ Departments of Neuroscience \& Physiology, NYU Langone Medical Center, New York, USA \\ ${ }^{3}$ Departments of Biochemistry \& Molecular Pharmacology, NYU Langone Medical Center, New York, USA \\ Email: *Alexandra.Sideris@nyumc.org
}

Received 10 July 2014; revised 17 August 2014; accepted 8 October 2014

Copyright (C) 2014 by authors and Scientific Research Publishing Inc.

This work is licensed under the Creative Commons Attribution International License (CC BY).

http://creativecommons.org/licenses/by/4.0/

(c) (i) Open Access

\begin{abstract}
The spared nerve injury (SNI) model of neuropathic pain was first developed by Decosterd and Woolf in 2000 in Sprague Dawley rats to enhance reproducibility of injury and behavioral responses resulting from a partial nerve injury. Given the differences in methodology and inconsistent behavioral data published in the SNI model of neuropathic pain in mice, and given that interspecies behavioral comparisons using the same peripheral nerve injury are presently lacking, in this study we assessed the development of mechanical and cold allodynia for five weeks in C57BL/6 mice and Sprague Dawley rats that underwent SNI. In rats and mice, the tibial and peroneal branches were ligated then severed, leaving the sural branch intact. By controlling several factors in the surgical procedure and behavioral tests, we found that rats developed and maintained strong mechanical and robust cold allodynia immediately following the injury that was maintained for the duration of the experiment (five weeks). In comparison, mice developed mechanical allodynia to a lesser magnitude which peaked at 2 weeks, but did not develop cold allodynia. We found both temporal and qualitative differences in the development of allodynic behaviors between SNI-mice and SNI-rats. Parallel analysis of interspecies differences can be exploited to reveal novel molecular players leading to divergent pain behaviors.
\end{abstract}

\section{Keywords}

SNI, Rats, Mice, Mechanical Allodynia, Cold Allodynia

\footnotetext{
"Corresponding author.
}

How to cite this paper: Sideris, A., Norcini, M., Blanck, T.J.J. and Recio-Pinto, E. (2014) Temporal and Qualitative Differences in the Development of Allodynic Behaviors between Mice and Rats in a Peripheral Nerve Injury Model. Pain Studies and Treatment, 2, 121-127. http://dx.doi.org/10.4236/pst.2014.24019 


\section{Introduction}

\section{Background}

Persistent post-operative pain (PPP) can be a consequence of nerve injury [1]. Genetic and psychosocial factors, pre-existing diseases, extent of iatrogenic nerve damage following surgery, and perioperative pain management contribute to the heterogeneity of patient outcomes with regards to the type, magnitude and duration of experienced pain [2]. Animal models allow for a systematic and well-controlled environment whereby each of these variables can be independently or concomitantly examined and are useful for identifying potential therapeutic targets for neuropathic pain. Even though both mice and rats are routinely used in pre-clinical pain models, interspecies comparisons using the same, established nerve injury model are scarce. SNI is advantageous over other partial nerve injury models (partial sciatic nerve ligation, chronic constriction injury, and spinal nerve ligation) because the level of injury is reproducible. It produces robust behavioral changes in rats, and affords the potential to study both injured and non-injured nerves and associated skin territories [3]. In rats, cold allodynia, heat hyperalgesia, mechanical allodynia and hyperalgesia develop immediately after SNI and are maintained for the observation period (ranging from 64 to 220 days) [3]. While in rats SNI consistently produces cold and mechanical allodynia in the ipsilateral paws [4]-[6], SNI in mice is not so consistent. Some studies show the development of mechanical hypersensitivity and others do not [7]-[9]. Given the importance and prevalence of transgenic approaches in pain research for which mice are readily used, the SNI model was extended to C57BL/6 mice [7] [8]. The choices of injury and rodent species/strain are important considerations in the study of allodynia in the pre-clinical setting as they both confer distinct advantages. However, reported inconsistencies within strain and within injury models become problematic.

The goals of this study were to induce the SNI in Sprague Dawley rats and C57BL/6 mice and systematically study the development of mechanical and cold allodynia. This was done in order to understand the extent to which species-specific differences affect the quality and chronicity of the pain-like behaviors that develop.

\section{Materials \& Methods}

All experiments were approved by and conducted in accordance with the guidelines set forth by the Institutional Animal Care and Usage Committee of NYU Langone Medical Center.

\subsection{Spared Nerve Injury Model}

Adult male Sprague Dawley rats (250 - $300 \mathrm{~g})$ and 4-month-old male C57BL/6 mice (25 - $30 \mathrm{~g})$ were subjected to SNI according to previously published studies, with slight modifications [3] [7] [8]. Using aseptic surgical techniques and isoflurane anesthesia, an incision $(\sim 0.8 \mathrm{~cm})$ was made in the skin of the right hindlimb to expose the muscle. The skin was detached from the muscle, and the sciatic nerve was identified by exposing the intermuscular space between the gluteus maximus and the anterior head of the bicep femoris. Muscles were not cut or excessively stretched. The connective tissues were carefully removed with fine forceps to loosen the branches from one another. In the mouse SNI, in an effort to minimize the stretching and agitation of the sural branch, we combined the approaches of the two seminal mouse studies: we used a very small (9-0) suture to ligate the tibial and peroneal branches together [7] [8] and removed a $2 \mathrm{~mm}$ nerve stump distal to the ligation. The muscle and skin were sutured separately, using 6-0 and 5-0 sutures, respectively. In the rat SNI, the peroneal and tibial branches were ligated separately using a 5-0 suture, the branches severed, and $2 \mathrm{~mm}$ stumps were removed distal to the ligations. The muscle and skin were sutured separately, using 4-0 and 3-0 sutures, respectively. In addition to avoiding agitation of the sural branch, care was taken to avoid tearing the blood vessels over the tibial and peroneal branches. Immediate post-operative recovery included a subcutaneous injection of saline, rest on a heating pad for 45 minutes, and access to food and water. For the duration of the experiments, animals were housed in separate cages. Because of the aseptic surgical technique, prophylactic or empirical antibiotic therapy was not necessary. Following the surgery, the incidence of autotomy was low $(<5 \%)$; those animals were not used.

\subsection{Behavioral Testing for Mechanical and Cold Allodynia}

Several days prior to collecting baseline thresholds, animals were handled and introduced to the behavior room to minimize anxiety. Pre- and post-injury, rats and mice were never tested in the same room, or using the same 
equipment, as these stressors can confound the behavioral data. On each test day, the von Frey test was conducted first, animals given approximately 30 minutes to rest, and then challenged with acetone. Each animal was stimulated 2 - 5 times in each hindpaw, with a rest period of approximately 5 minutes between each stimulation.

\subsection{Electronic von Frey}

Prior to SNI, baseline mechanical thresholds were assessed in both hindpaws using the Electronic von Frey apparatus (IITC Life Sciences, Inc.): for rats, a size 15 filament was fitted on the 800 gram arm, and for mice, a size 8 filament fitted on a 90 gram arm. Changes in the weight supported by each paw were measured for 5 weeks following injury. Significant decreases in threshold, relative to baseline, indicated the development of mechanical allodynia.

\subsection{Acetone Test}

Cold allodynia was assessed by using a modified acetone test [10]. The duration of paw withdrawal in the 30 seconds immediately following acetone application to the plantar surface of the hindpaws was measured to assess cold allodynia at baseline a following SNI. Absolute acetone $(100 \%)$ in a volume of $20 \mu$ ls was placed on the base of the paw for rats, while $2 \mu \mathrm{ls}$ for mice. During acetone application the pipette tips did not touch the paw. Oneway ANOVAs were used to determine significance within species, and unpaired student's t-test used to determine significance between species per time point.

\section{Results}

\subsection{After SNI the Magnitude and Onset of Mechanical Allodynia Differs in Mice and Rats}

Rats developed statistically significant mechanical allodynia ( $70 \%$ decrease in threshold relative to baseline) in the ipsilateral paw 1 day post-SNI $(p<0.001)$, which was maintained for the 5 week observation period. Mice also developed mechanical allodynia immediately after the injury. However, mice displayed only a $30 \%$ decrease in threshold relative to baseline at POD 1 (Figure 1). Though mechanical allodynia peaked at POD 1 and was maintained until POD 35 in rats, it took approximately 2 weeks for mechanical allodynia to peak in mice (POD 14). In mice, the peak was a $50 \%$ decrease in threshold relative to baseline (versus a $70 \%$ decrease in rats) that was maintained for the remaining 3 weeks (Figure 1).

\subsection{Cold Allodynia Develops in Rats but Not Mice after SNI}

Interestingly, though both species developed mechanical allodynia following SNI, only rats developed robust cold allodynia in the ipsilateral paw, which peaked at 3 weeks (POD 21) and was maintained until the 5th week (Figure 2). The contralateral paws did not develop cold allodynia.

\section{Discussion}

There are immense strengths in the use of rodents for studying neuropathic pain, and SNI has many advantages. There is little variation in the magnitude of the injury because the same two branches are ligated and severed. The reproducibility of injury affords the potential to study both injured and non-injured nerves in a consistent manner. Table 1 details specific methodologies used in the three seminal SNI studies. Our data show that when the level of injury and anesthesia are systematically controlled in the SNI model, both mice and rats develop mechanical allodynia. This behavior varies in magnitude and the onset displays a different time course. In addition, rats developed robust cold allodynia, while mice did not.

The previously reported inconsistencies in behavioral responses in the mouse SNI model, even when using the same strain, become problematic because mice are readily used for transgenic approaches [11]. It is not clear why SNI in C57Bl/6 mice does not induce mechanical allodynia in some studies, yet it does in others [7] [8]. It may be due to differences in the anesthesia used during surgery, the size of suture used for ligation, or other details of the surgical procedure and post-op recovery period. Anesthesia type may not be the strongest factor for the observed differences because even though the first mouse SNI study indicates that the use of Ketamine and Xylazine precludes the development of mechanical hypersensitivity [7], several studies have used this exact cocktail and showed that mechanical hyperalgesia and allodynia develop immediately after the injury in 


\begin{tabular}{|c|c|c|}
\hline Electronic von Frey & Mice & Rats \\
\hline Acclimatization & 45 minutes & 45 minutes \\
\hline Stimulating arm & 90 gram arm & 800 gram arm \\
\hline Filament & Size 8 & Size 15 \\
\hline Area of stimulation & $\begin{array}{c}\text { Sural area of } \\
\text { hindpaws } \\
\text { (lateral/plantar } \\
\text { surface) }\end{array}$ & $\begin{array}{c}\text { Sural area of } \\
\text { hindpaws } \\
\text { (lateral/plantar } \\
\text { surface) }\end{array}$ \\
\hline Readout & $\begin{array}{c}\text { Maximum weight } \\
\text { supported by paw }\end{array}$ & $\begin{array}{c}\text { Maximum weight } \\
\text { supported by paw }\end{array}$ \\
\hline stimulations & 5 minutes & 5 minutes \\
\hline
\end{tabular}

(a)

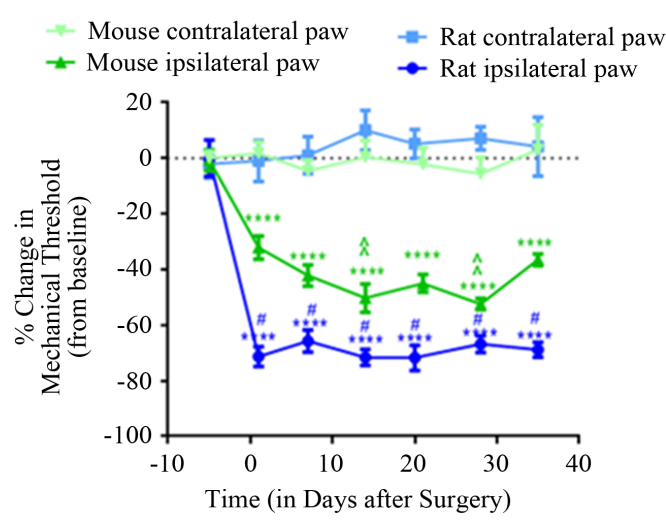

(b)

Figure 1. Development of mechanical allodynia in C57BL/6 mice and Sprague Dawley rats following SNI: (a) Comparison of methods used for Electronic von Frey; (b) Changes in mechanical threshold following SNI in the contralateral (light green) and ipsilateral hindpaws (dark green) of mice, $\mathrm{N}=11$, and in the contralateral (light blue) and ipsilateral (dark blue) hindpaws of rats, $\mathrm{N}=10$, are expressed as a percentage relative to baseline. Mechanical allodynia-ipsilateral paw-in mice (bold green) peaks later, and to a lesser degree compared to rats (bold blue). $p<0.001$ versus baseline, $p<0.05$ rat versus mouse, $p<0.05$ versus mouse post-op Day 1 . At all post-surgery time points, the responses in the ipsilateral paws were significantly different from their corresponding contralateral paws, in both mice and rats $(p<0.001)$ (not indicated in graph).

\begin{tabular}{|c|c|c|}
\hline Acetone Test & Mice & Rats \\
\hline Volume & $2 \mu \mathrm{ls}$ & $20 \mu \mathrm{ls}$ \\
\hline Pipette & Pipetman & Stepper/Repeating \\
\hline Measurement & $\begin{array}{c}\text { Paw withdrawal } \\
\text { duration } \\
(30 \text { seconds })\end{array}$ & $\begin{array}{c}\text { Paw withdrawal } \\
\text { duration } \\
(30 \text { seconds })\end{array}$ \\
\hline $\begin{array}{c}\text { Time between } \\
\text { stimulations }\end{array}$ & $\sim 5$ minutes & $\sim 5$ minutes \\
\hline
\end{tabular}

(a)

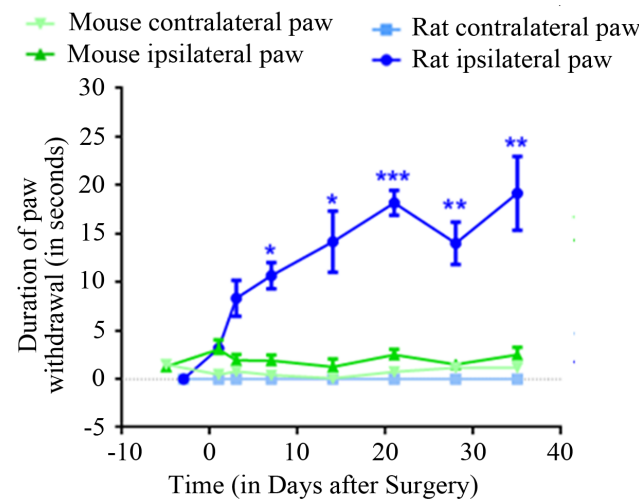

(b)

Figure 2. Development of cold allodynia using the acetone drop test in C57BL/6 mice and Sprague Dawley rats following SNI: (a) Comparison of methods used for the Acetone drop test; (b) Baseline and post-SNI acetone responses in the contralateral (light green) and ipsilateral hindpaws (dark green) of mice, $\mathrm{N}=9$, and in the contralateral (light blue) and ipsilateral (dark blue) hindpaws of rats, $\mathrm{N}=3$, expressed in seconds of hindpaw withdrawal. The day that SNI surgery was performed is indicated as Day 0. Cold allodynia never develops in mice (dark green) but develops in rats (blue). $p<0.05, p<0.01, p<0.001$ versus baseline.

C57BL/6 mice [12] [13]. In the acetone test for cold allodynia, the majority of papers either do not define a particular volume that is applied to the paws, or report a range of volumes, making reproducibility difficult [14][16]. Care must be taken in the acetone drop test because there may be a mechanical stimulation component if syringe tips touch the paws, or if the "spray" of acetone is too forceful. Additionally, it has previously been documented that the acetone test produces an uncontrollable global behavior on the mouse that severely impairs an accurate recording of behaviors [17]. The droplet volume chosen in the present study was based on our observation that acetone volumes greater than $5 \mu$ ls produced prolonged paw withdrawals in non-injured animals before surgery (data not shown). Reducing the volume to $2 \mu \mathrm{ls}$, and carefully placing this droplet on the plantar surface of the mouse's hindpaws induced a baseline withdrawal duration of approximately 2 seconds in normal, wildtype mice. A withdrawal duration of 2 seconds is also seen with $2 \mu \mathrm{ls}$ of room temperature water at baseline. This acetone volume, however, is sufficient to induce a hypersensitivity response under other conditions - data previously published in abstract form [18]. 
Table 1. Comparison of sural Spared Nerve injury models in rats and mice including strain, gender of rodents, weight, age, type of anesthesia used, side of injury, how the injury was induced, suture sizes, and behaviors measured after the injury.

\begin{tabular}{|c|c|c|c|}
\hline & Decosterd \& Woolf 2000 & Shields et al. 2003 & Bourquin et al. 2006 \\
\hline Species/Strain & Sprague Dawley Rats & $\mathrm{C} 57 \mathrm{BL} / 6$ mice & $\mathrm{C} 57 \mathrm{BL} / 6$ mice \\
\hline Gender & Male & Male \& Female & Male \\
\hline Weight/Age & $200-250 \mathrm{~g}$ (age not specified) & $20-25 \mathrm{~g}$ (age not specified) & $25-30 \mathrm{~g} / 2$ months old \\
\hline Anesthesia & Halothane $(2 \%)$ & $\begin{array}{c}\text { Ketamine \& Xylazine } \\
(60 \text { and } 8 \mathrm{mg} / \mathrm{kg} \text { ) OR Halothane } \\
(1.75 \%-2.5 \%)\end{array}$ & Isoflurane $(1.5 \%-2.5 \%)$ \\
\hline \multirow[t]{2}{*}{ Side of Injury } & Not specified & Not specified & Left hindlimb \\
\hline & $\begin{array}{l}\text { Tibial ligated/peroneal ligated; } \\
\text { both severed, 2-4mm stumps } \\
\text { removed distal to ligations }\end{array}$ & $\begin{array}{c}\text { Tibial ligated/peroneal ligated; } \\
\text { both severed }\end{array}$ & $\begin{array}{l}\text { Tibial + peroneal ligated together; } \\
1 \text { - } 2 \text { mm stumps removed } \\
\text { distal to ligation }\end{array}$ \\
\hline \multicolumn{4}{|l|}{ Injury } \\
\hline Nerve Ligation & 5-0 silk & $9-0$ silk & 6-0 silk \\
\hline Muscle Suture & Not specified & 6-0 silk & $6-0$ silk \\
\hline Skin Suture & Not specified & 6-0 silk & 6-0 silk \& surgical micro clips \\
\hline Mechanical Allodynia & Yes & $\begin{array}{l}\text { Not with Ketamine/Xylazine; } \\
\text { Not with Halothane }\end{array}$ & Yes \\
\hline Cold Allodynia & Yes & Not determined & Not determined \\
\hline
\end{tabular}

\section{Conclusions}

A multi-model approach is necessary to study the mechanisms of neuropathic pain, but it is usually reserved for examining differences in various peripheral nerve injury models within the same species [3] [7] [19], or for examining the same injury model in different strains of the same species [20] [21]. In the pain literature, results from rats and mice are oftentimes grouped into "rodent" models, and subtle differences are not acknowledged. We argue that interspecies comparisons between rodents also have a place in translational research.

Compared with mice, our results indicate that SNI in rats induces a more severe evoked-pain phenotype, characterized by immediate strong mechanical allodynia and robust, yet delayed, cold allodynia. We further propose that SNI in mice may be a negative model for nerve-injury induced cold allodynia. Ultimately, the utility of these pre-clinical models is best realized through "back-translation" in which patient phenotypes can inform and refine the rodent paradigms. The appropriate animal models can be chosen on the basis of specific patient phenotypes in order to increase predictive validity of therapeutic interventions for a particular pain condition. For example, cold allodynia is not present in all patients who have peripheral nerve injury-induced mechanical allodynia [22], and it has been proposed that cold allodynia may be a hallmark of sympathetically maintained pain [23]. The use of these two species would help discern what factors (e.g. peripherally or centrally derived) may be contributing to differences in the development of cold allodynia following a peripheral nerve injury. Furthermore, since there are numerous reports emphasizing the genetic component of pain [24], it will be important to detail the precise nature and extent of behavioral similarities between rats and mice with SNI because genetic targets for neuropathic pain remain elusive [25]. Hence, parallel analysis of interspecies differences can be exploited to reveal novel molecular players leading to divergent pain behaviors. These approaches may facilitate the human translational process by refining target validation at the pre-clinical level. 


\section{Competing Interests}

The authors declare that they have no competing interests.

\section{Authors' Contributions}

All authors read and approved the final manuscript. AS and MN conducted the experiments and analyzed the data. AS wrote the manuscript. TJJB and ERP were involved with study design and editing of the manuscript.

\section{Acknowledgements}

This work was completed with support from the NYU Langone Medical Center Department of Anesthesiology Research Funds. The authors wish to thank: Dr. Jin Zhang, Lourdes Martin Alejandra Hernandez, and Puja Bhardwaj for helping with the collection of rat behavioral data; Dr. Brian Schmidt and members of his laboratory for assisting with setting up the von Frey apparatus for mice; Dr. Lori Russo, Joyce Chen and Shanelle Mason for assisting with surgeries and the collection of behavioral data from the mice. Special thanks to Dr. Lisa Doan for helpful discussions.

\section{References}

[1] Macrae, W.A. (2001) Chronic Pain after Surgery. British Journal of Anaesthesia, 87, 88-98. http://dx.doi.org/10.1093/bja/87.1.88

[2] Johansen, A., et al. (2012) Persistent Postsurgical Pain in a General Population: Prevalence and Predictors in the Tromso Study. Pain, 153, 1390-1396. http://dx.doi.org/10.1016/i.pain.2012.02.018

[3] Decosterd, I. and Woolf, C.J. (2000) Spared Nerve Injury: An Animal Model of Persistent Peripheral Neuropathic Pain. Pain, 87, 149-158. http://dx.doi.org/10.1016/S0304-3959(00)00276-1

[4] Yang, F., et al. (2013) Analgesic Treatment with Pregabalin Does Not Prevent Persistent Pain after Peripheral Nerve Injury in the Rat. Pain, 155, 356-366.

[5] Wang, J., et al. (2011) A Single Subanesthetic Dose of Ketamine Relieves Depression-Like Behaviors Induced by Neuropathic Pain in Rats. Anesthesiology, 115, 812-821. http://dx.doi.org/10.1097/ALN.0b013e31822f16ae

[6] Swartjes, M., et al. (2011) Nonselective and NR2B-Selective N-Methyl-D-Aspartic Acid Receptor Antagonists Produce Antinociception and Long-Term Relief of Allodynia in Acute and Neuropathic Pain. Anesthesiology, 115, 165174. http://dx.doi.org/10.1097/ALN.0b013e31821bdb9b

[7] Shields, S.D., Eckert III, W.A and Basbaum, A.I. (2003) Spared Nerve Injury Model of Neuropathic Pain in the Mouse: A Behavioral and Anatomic Analysis. The Journal of Pain, 4, 465-470. http://dx.doi.org/10.1067/S1526-5900(03)00781-8

[8] Bourquin, A.F., et al. (2006) Assessment and Analysis of Mechanical Allodynia-Like Behavior Induced by Spared Nerve Injury (SNI) in the Mouse. Pain, 122, 14e1-14.

[9] Abe, K., Fujii, Y. and Nojima, H. (2011) Evaluation of Hyperalgesia in Spared Nerve Injury Model Using Mechanical, Thermal, and Chemical Stimuli in the Mouse. Neurological Research, 33, 656-662. http://dx.doi.org/10.1179/1743132810Y.0000000019

[10] Choi, Y., et al. (1994) Behavioral Signs of Ongoing Pain and Cold Allodynia in a Rat Model of Neuropathic Pain. Pain, 59, 369-376. http://dx.doi.org/10.1016/0304-3959(94)90023-X

[11] Hau, J. (2008) Animal Models for Human Diseases. In: Conn, P.M., Ed., Sourcebook of Models for Biomedical Research, Humana Press, New York, 3-8. http://dx.doi.org/10.1007/978-1-59745-285-4 1

[12] Richner, M., Bjerrum, O.J., Nykjaer, A. and Vaegter, C.B. (2011) The Spared Nerve Injury (SNI) Model of Induced Mechanical Allodynia in Mice. Journal of Visualized Experiments, 54, e3092.

[13] Smith, A.K., O’Hara, C.L. and Stucky, C.L. (2013) Mechanical Sensitization of Cutaneous Sensory Fibers in the Spared Nerve Injury Mouse Model. Molecular Pain, 9, 61. http://dx.doi.org/10.1186/1744-8069-9-61

[14] Kuhlein, H.N., Tegeder, I., Möser, C., Lim, H.-Y., et al. (2011) Nerve Injury Evoked Loss of Latexin Expression in Spinal Cord Neurons Contributes to the Development of Neuropathic Pain. PLoS ONE, 6, e19270. http://dx.doi.org/10.1371/journal.pone.0019270

[15] Vachon, P., Millecamps, M., Low, L., Thompsosn, S.J., Pailleux, F., et al. (2013) Alleviation of Chronic Neuropathic Pain by Environmental Enrichment in Mice Well after the Establishment of Chronic Pain. Behavioral and Brain Functions, 9, 22. http://dx.doi.org/10.1186/1744-9081-9-22 
[16] Solway, B., Bose, S.C., Corder, G., Donahue, R.R. and Taylor, B.K. (2011) Tonic Inhibition of Chronic Pain by Neuropeptide Y. Proceedings of the National Academy of Sciences of the United States of America, 108, 7224-7229. http://dx.doi.org/10.1073/pnas.1017719108

[17] Pertin, M., Gosselin, R.D. and Decosterd, I. (2012) The Spared Nerve Injury Model of Neuropathic Pain. Methods in Molecular Biology, 851, 205-212. http://dx.doi.org/10.1007/978-1-61779-561-9 15

[18] Sideris, A.N.M., Russo, L., Chen, J., Mason, S., Blanck, T.J.J. and Recio-Pinto, E. (2013) Knock out of the Cannabinoid 1 Receptor Promotes Recovery from Mechanical but Not from Cold Allodynia Following Sural Spared Nerve Injury in Mice. Neuropathic Pain Special Interest Group Congress, Toronto.

[19] Kim, K.J., Yoon, Y.W. and Chung, J.M. (1997) Comparison of Three Rodent Neuropathic Pain Models. Experimental Brain Research, 113, 200-206. http://dx.doi.org/10.1007/BF02450318

[20] Persson, A.-K., Xu, X.-J., Wiesenfeld-Hallin, Z., Devor, M. and Fried, K. (2010) Expression of DRG Candidate Pain Molecules after Nerve Injury-A Comparative Study among Five Inbred Mouse Strains with Contrasting Pain Phenotypes. Journal of the Peripheral Nervous System, 15, 26-39. http://dx.doi.org/10.1111/j.1529-8027.2010.00249.x

[21] Xu, X.-J., Plesan, A., Yu, W., Hao, J.-X. and Wiesenfeld-Hallin, Z. (2001) Possible Impact of Genetic Differences on the Development of Neuropathic Pain-Like Behaviors after Unilateral Sciatic Nerve Ischemic Injury in Rats. Pain, 89, 135-145. http://dx.doi.org/10.1016/S0304-3959(00)00356-0

[22] Gierthmuhlen, J., Maier, C., Baron, R., Tölle, T., Treede, R.-D., et al. (2012) Sensory Signs in Complex Regional Pain Syndrome and Peripheral Nerve Injury. Pain, 153, 765-774. http://dx.doi.org/10.1016/j.pain.2011.11.009

[23] Zhao, C.S., Chen, L., Tao, Y.-X., Tall, J.M., et al. (2007) Lumbar Sympathectomy Attenuates Cold Allodynia but Not Mechanical Allodynia and Hyperalgesia in Rats with Spared Nerve Injury. The Journal of Pain, 8, 931-937. http://dx.doi.org/10.1016/i.jpain.2007.06.008

[24] Young, E.E., Lariviere, W.R. and Belfer, I. (2012) Genetic Basis of Pain Variability: Recent Advances. Journal of Medical Genetics, 49, 1-9. http://dx.doi.org/10.1136/jmedgenet-2011-100386

[25] Kim, H., Clark, D. and Dionne, R.A. (2009) Genetic Contributions to Clinical Pain and Analgesia: Avoiding Pitfalls in Genetic Research. The Journal of Pain, 10, 663-693. http://dx.doi.org/10.1016/j.jpain.2009.04.001

\section{Abbreviations}

SNI: spared nerve injury;

POD: post-operative day. 
Scientific Research Publishing (SCIRP) is one of the largest Open Access journal publishers. It is currently publishing more than 200 open access, online, peer-reviewed journals covering a wide range of academic disciplines. SCIRP serves the worldwide academic communities and contributes to the progress and application of science with its publication.

Other selected journals from SCIRP are listed as below. Submit your manuscript to us via either submit@scirp.org or Online Submission Portal.
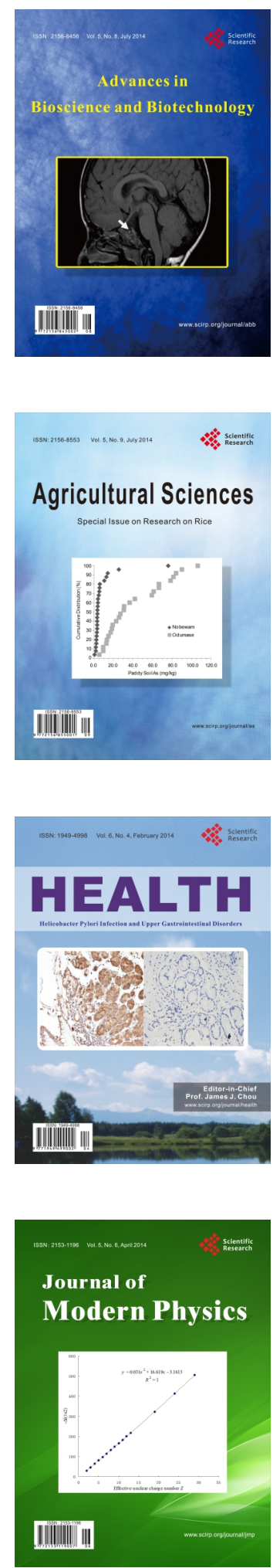
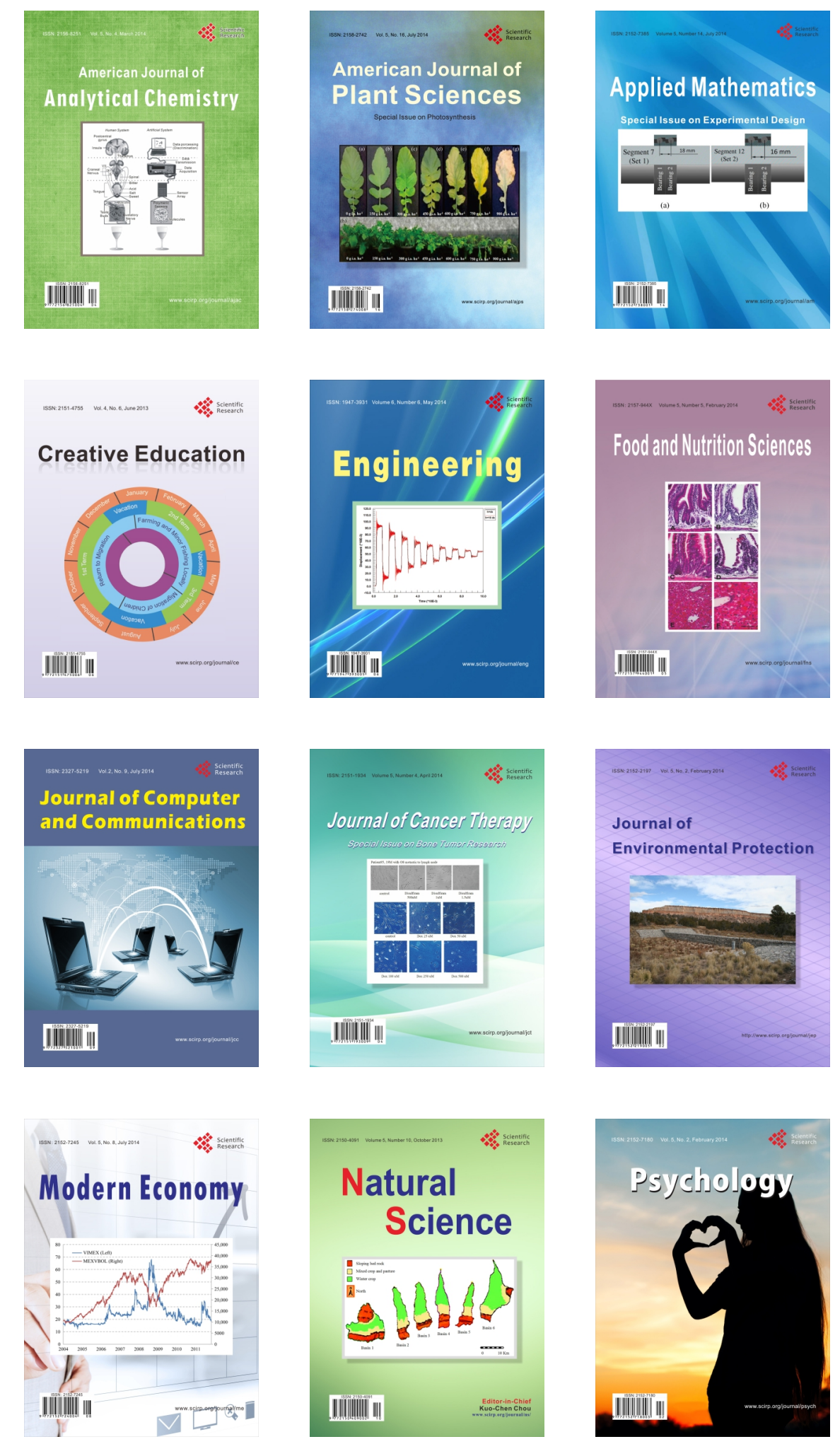